\title{
Genetic Exchange of Lung-Derived Exosome to Brain Causing Neuronal Changes on COVID-19 Infection
}

\author{
Shiek S. S. J. Ahmed ${ }^{1}$ (I) Prabu Paramasivam² Manjunath Kamath $^{3} \cdot$ Ashutosh Sharma $^{4} \cdot$ Sophie Rome ${ }^{5}$. \\ Ram Murugesan ${ }^{1}$
}

Received: 20 January 2021 / Accepted: 7 July 2021 / Published online: 27 July 2021

(c) The Author(s), under exclusive licence to Springer Science+Business Media, LLC, part of Springer Nature 2021

\begin{abstract}
The pandemic of novel coronavirus 2 (SARS-CoV-2) has made global chaos for normal human living. Despite common COVID-19 symptoms, variability in clinical phenotypes was reported worldwide. Reports on SARS-CoV-2 suggest causing neurological manifestation. In addition, the susceptibility of SARS-CoV-2 in patients with neurodegenerative diseases and its complexity are largely unclear. Here, we aimed to demonstrate the possible transport of exosome from SARS-CoV-2infected lungs to the brain regions associated with neurodegenerative diseases using multiple transcriptome datasets of SARS-CoV-2-infected lungs, RNA profiles from lung exosome, and gene expression profiles of the human brain. Upon transport, the transcription factors localized in the exosome regulate genes at lateral substantia nigra, medial substantia nigra, and superior frontal gyrus regions of Parkinson's disease (PD) and frontal cortex, hippocampus, and temporal cortex of Alzheimer's disease (AD). On SARS-CoV-2 infection, BCL3, JUND, MXD1, IRF2, IRF9, and STAT1 transcription factors in the exosomes influence the neuronal gene regulatory network and accelerate neurodegeneration. STAT1 transcription factor regulates 64 PD genes at lateral substantia nigra, 65 at superior frontal gyrus, and 19 at medial substantia nigra. Similarly, in AD, STAT1 regulates 74 AD genes at the temporal cortex, 40 genes at the hippocampus, and 16 genes at the frontal cortex. We further demonstrate that dysregulated neuronal genes showed involvement in immune response, signal transduction, apoptosis, and stress response process. In conclusion, SARS-CoV-2 may dysregulate neuronal gene regulatory network through exosomes that attenuate disease severity of neurodegeneration.
\end{abstract}

Keywords SARS-CoV-2 $\cdot$ Covid-19 $\cdot$ Exosome $\cdot$ Neurodegeneration $\cdot$ Parkinson's disease $\cdot$ Alzheimer's disease

Shiek S. S. J. Ahmed

shiekssjahmed@gmail.com

1 Omics and Drug Discovery Lab, Faculty of Allied Health Sciences, Chettinad Academy of Research and Education, Kelambakkam, Tamil Nadu 603103, India

2 Department of Neurology, School of Medicine, University of New Mexico Health Sciences Center, University of New Mexico, Albuquerque, NM, USA

3 Department of Pharmacology, Saveetha Dental College, Saveetha Institute of Medical and Technical Sciences, Chennai, Tamil Nadu, India

4 Tecnologico de Monterrey, School of Engineering and Sciences, Campus Querétaro, Santiago de Querétaro, Mexico

5 CarMeNlaboratory, Cardiovasculaire, Métabolisme, Diabétologie Et Nutrition, University of Lyon, Lyon, France

\section{Introduction}

Exosomes are one of the extracellular vesicles secreted by most of the multi-cellular organisms for their intercellular communication. Exosomes carry various biomolecules like nucleotide molecules, including RNA, miRNAs, IncRNA, proteins, and metabolites from the originating cells to target cells $[1,2]$. Initially, exosomes were considered to be involved in removing waste molecules from the cells [3]. Advance in the research suggests that exosomes deliver micro- and macromolecules to communicate and regulate the recipient cells during physiological and pathological processes. It is proven that exosomes are involved in cancer progression [4], cardiovascular diseases [5], neurodegeneration [6], and even in microbial and viral infections [7, 8]. Interestingly, exosomes released from the virus-infected cells carry the viral particles to reprogram the target cells and spread pathogenesis [9]. 
Recently, the SARS-CoV-2 (COVID-19) infection has become a pandemic affecting millions of people worldwide. An increased vulnerability is reported in patients with chronic diseases like diabetes and hypertension [10, 11]. SARS-CoV-2 infects lower respiratory tracts and progresses toward multi-organ failure [12]. Currently, there is no specific treatment available to treat COVID-19. Social distancing and vaccination are helpful to protect the spreading of disease among the population. However, there is an urgent need to develop a drug to treat this disease. Understanding the disease mechanism may contribute to the development of a potential drug for SARS-CoV-2 infection. Many viruses, including the RNA of SARS-CoV-2, are known to enter the exosome that enables intra-host spreading [13]. Studies showed presence of SARS-CoV-2 viral particles within exosomes [14, 15]. Besides, SARS-CoV-2 was noticed within host cells' vacuoles in the histopathological analysis $[16,17]$. These findings suggested a possible involvement of exosomal intercellular communication on SARS-CoV-2 infection from the primary lung site to other communicating organs in the human system.

Among various affected organs, limited research has been carried out to study the COVID-19 pathogenesis in the brain. Besides the cardinal symptoms, neurological changes have also been reported in $36.4 \%$ of cases COVID-19 [18]. Notably, some COVID-19 recovered patients show significant memory loss and cognitive disability [19]. Recently, Singh et al. suggested that COVID-19 may affect the central nervous system (CNS) [20]. Particularly, a few studies suggest COVID-19 may accelerate neurodegeneration in Parkinson's (PD) and Alzheimer's (AD) disease [21, 22]. A study by Pavel et al. suggests COVID-19 causes selective vulnerability in PD by activation of $\alpha$-synucleinopathies in the CNS [21]. Also, the survey conducted by Brown et al. reports the worsening of PD symptoms on COVID-19 infection [23]. Similarly, Naughton et al. demonstrate the possible role of COVID-19 infection in patients with AD [24]. Moreover, Heneka et al. suggest that COVID-19 patients are at high risk of developing $\mathrm{AD}$ [25].

Herein, we postulate that exosomes may be involved in the transport molecules from the infected lungs to the brain region by crossing the blood-brain barrier (BBB) that leads to neurological manifestation. Elevated levels of exosomes in the peripheral circulatory system were noticed during lung inflammation [26]. Indeed, previous studies have demonstrated the exosomal transport between the lungs to BBB and BBB to other neuronal regions [27-32]. In general, BBB is a vascular structure composed of brain microvascular endothelial cells (BMECs), which act as a barrier between the CNS and peripheral circulatory system that allows exosomes in and out from blood to the brain. Studies suggest exosomal transport occurs by endocytosis of BMEC's transcellular mechanism or diffusion through tight junctions between BMECs [33, 34]. Recently, Reynolds et al. suggest the ability of SARS-COV-2 to alter tight junction (TJ) proteins that cause disruption of BBB integrity which allows neuro-invasion of molecules [35]. Chen et al. demonstrate exosomes crossing BBB through transcellular BMEC endocytosis in stroke [36]. Similarly, Matsumoto et al. report the transport of exosomes from the peripheral circulatory to CNS through BBB during the inflammatory condition [37].

In the present study, we have developed a systems biological framework to demonstrate the role of exosomecarried mRNAs that encode transcription factors from infected lungs to the brain. We investigated multiple transcriptome datasets of SARS-CoV-2-infected lungs, RNA profiles of lung-derived exosomes, and gene expression profiles of various human brain regions. In addition, the exosomal intercellular network between lungs and brain regions was constructed for neurodegenerative diseases based on literature evidence. Our data identify the transcription factors (TF) from the lungs regulating gene expression in the brain regions, which accelerate $\mathrm{PD}$ and AD on COVID-19 infection.

\section{Materials and Methods}

\section{Data Collection}

The RNA-Seq dataset related to SARS-CoV-2 infection in the lungs were retrieved from Sequence Read Archive (SRA), NCBI database by using a combination of key terms including "SARS-CoV-2" and "Human host." The dataset (GSE147507) containing the RNA-Seq profile of 110 samples at various experimental conditions of SARS-CoV-2 infection was retrieved [38]. Among 110 samples, two healthy lung biopsies (GSM4462413 and GSM4462414) and two SARS-CoV-2-infected lung (GSM4462415 and GSM4462416) samples were selected and utilized for our downstream analysis. These selected samples have no previous history of disease including neuropathology.

\section{RNA-Seq Expression Analysis}

FastQC v0.11.5 was used to assess the quality of data. Then, reads were trimmed by using Trimmomatic v0.32. The qualified reads were aligned to the HG19 human genome. Reads count for each transcript was normalized, and the up-regulated genes on SARS-CoV-2 infection compared to healthy lungs were captured using the DESeq method. Significant up-regulated genes were identified based on false discovery rate (FDR), $p<0.05$. 


\section{Lung Exosome Profile}

The GSE121307 microarray dataset [39] describing the exosomal shuttle RNA (esRNA) in respiratory tract-derived exosomes was collected from NCBI, Gene Expression Omnibus (GEO) database. Exosomal shuttle mRNA (esRNA) predominantly expressed in GSE121307 lung data were identified and mapped with the overexpressed genes on SARS-CoV-2 infection. The mapped overexpressed genes were assumed to be packed into exosome as esRNA during SARS-CoV-2 infection. Among them, mRNA encoding transcription factor was identified using the Molecular Signatures (https://www.gsea-msigdb.org/gsea/msigdb) and TcoF-DB (https://tools.sschmeier.com/tcof) databases.

\section{Exosome Communication Network from Literature Data}

The Qinsight text mining tool (https://quertle.com/) was used to construct an exosome-cell communication network. Qinsight allows us to collect more precise literature for the key terms from patents, clinical trials, and journals, including original research, reviews, and case reports based on biospecific artificial intelligence methods. We constructed the exosome communication network by literature search with the relevant keywords to establish the exosome connection between (1) lungs to the BBB and (2) BBB to the neuronal regions associated to $\mathrm{PD}$ and $\mathrm{AD}$. At the initial phase of exosome communication network construction, anatomical neuronal connectivity between the brain regions and $\mathrm{BBB}$ was verified. In the second phase, the report suggesting the transport of exosomes between the lungs and the BBB and subsequent transfer of exosomes from the BBB to neuronal regions was accessed. In both phases, two different investigators (M.K. and P.P.) independently collected the supporting literature using Qinsight. The collected data were cross-verified by the two investigators (S.S.S.J.A. and A.S.). If any, discrepancy in the collected data was resolved during group discussion. Finally, based on the literature evidence, an exosome communication network was constructed.

\section{Neurodegenerative Disease Expression Profile}

The Parkinson's gene expression microarray dataset of human lateral substantia nigra, medial substantia nigra, and superior frontal gyrus regions was retrieved from the Gene Expression Omnibus (GSE8397) [40]. For AD, the gene expression dataset of human frontal cortex, hippocampus, and temporal cortex was collected with accession GSE36980 [41]. The sample characteristics of both datasets (GSE8397 and GSE36980) were assessed from the literature [40, 41]. In the PD dataset (GSE8397), all control samples were reported free from neurological diseases and PD cases were assessed by neurological rating scale and histologically confirmed [40]. Particularly, the multiple sclerosis-specific control sample in the GSE8397 dataset was excluded during analysis. Similarly in the AD dataset (GSE36980), Binswanger's disease and non-AD vascular dementia were excluded to select control samples that are free from neurological diseases [41], whereas the AD samples were pathologically assessed based on the Consortium to Establish a Registry for Alzheimer's Disease (CERAD) and the Braak stage positive. In addition, the $\mathrm{AD}$ was confirmed with immunostaining against phosphorylated microtubule-associated protein tau [41]. All selected samples from the datasets were log-transformed and analyzed with a limma 3.26.8 library package using the $\mathrm{R}$ program to identify significantly overexpressed genes (DEG) with a $t$ test statistical significance of $p \leq 0.05$.

\section{TF Regulating Genes}

Next, we assumed that exosome-transported mRNA encoding $\mathrm{TF}$ would be readily translated into protein by the host translational machinery and activate its target genes in neurons. These activated genes may be associated with neurodegenerative diseases. We use the Coexpedia database (http://www.coexpedia.org/) to look for overexpressed PD and $\mathrm{AD}$ genes that are co-expressed with each TF identified in the exosomes during SARS-CoV-2 infection. The regulatory relationships were determined between these TFs with overexpressed $\mathrm{PD}$ and $\mathrm{AD}$ genes using iRegulon plugin (version 1.3) in Cytoscape software [42] with NEScore $>3$ and FDR $<0.001$ set as a threshold [43].

\section{Molecular Enrichment}

Molecular enrichments were carried out to evaluate the TF's target genes' functions and relevance for the PD and AD pathology. The biological process of the genes in the regulatory network was determined by using the BiNGO module [44] with a $p$ value $<0.05$ cut-off in Cytoscape software. The regulatory network was also analyzed based on molecular pathways derived using the Reactome database (www.react ome.org/).

\section{Results}

\section{RNA-Seq Analysis and esRNA}

Lung RNA-Seq data on SARS-CoV-2 infection and their controls were analyzed. Of 21,797 transcripts, 731 (431 up and 300 down) were differentially regulated on SARS-CoV-2 infection with FDR, $p<0.05$. We then mapped the 431 overexpressed genes with GSE121307 esRNA data to confirm their lung-derived exosome. We believe that the flooding of 
overexpressed genes in the lungs in SARS-CoV-2 infection may be packed into exosomes and transported to the target cell. Among 431 overexpressed genes, 267 were sorted into exosomes. For example, GSK3B, DPP4, SMAD3, PARP1, and IKBKB are the inflammatory genes (mRNA) noticed as an outcome of cytokine storm in the lungs that may be carried as esRNA to target cells by the exosome. Of 267 overexpressed esRNA, 19 encode TFs identified using the Molecular Signatures and TcoF-DB v2 databases (Table 1).

\section{Exosomal Communication Network}

Next, we constructed an exosomal communication network based on literature evidence. The network demonstrates the exosomal connectivity between the lungs and BBB microendothelial cells [27-32]. Similarly, the exosome communication between the BBB endothelial cells and various neuronal regions was determined based on literature evidence [27-32]. Overall, the exosomal network confirms the exosomal connectivity between lungs and neuronal regions associated with neurodegenerative diseases through BBB (Fig. 1).

\section{Over-representation of Genes in Neurological Diseases}

Simultaneously, the collected microarray datasets of PD at lateral substantia nigra, medial substantia nigra, and superior frontal gyrus region were analyzed to identify the overexpressed genes at each neuronal region compared to its control. The overexpressed genes with a $p$ value $\leq 0.05$ were selected at each condition across the neuronal regions. For instance, 2495 genes at the lateral substantia nigra, 1088 at the medial substantia nigra, and 790 at the superior frontal gyrus region of PD were overexpressed compared to control. Similarly, the analysis of AD datasets showed 362 genes at the frontal cortex, 1935 in the hippocampus, and 1242 at the temporal cortex were noticed overexpressed in AD.

\section{TF-Gene Regulatory Network}

Transcription factor is a regulatory component involved in regulating cellular gene expression. Here, we built a TF-gene network for $19 \mathrm{TFs}$ regulating the overexpressed $\mathrm{PD}$ and $\mathrm{AD}$ genes across the brain regions. The acquired TFs from the exosomes will be readily translated into protein by the neuronal translational machinery of targeted neurons. After that, the TFs initiate the transcription of their target genes on binding to their promoter region. We noticed 2758 genes were co-expressed along with the selected 19 TFs (Fig. 2). Using iRegulon, the six transcription factors showed regulating the overexpressed genes in neurodegenerative diseases at lateral substantia nigra (Fig. 3), medial substantia nigra (Fig. 4), superior frontal gyrus (Fig. 5), frontal cortex (Fig. 6), hippocampus (Fig. 7), and temporal cortex (Fig. 8). For instance, STAT1 regulates 64 genes associated with PD at lateral substantia nigra. In medial

Table 1 Transcription factors localized into lung-derived exosome on COVID infection

\begin{tabular}{|c|c|c|}
\hline Transcription factor & Description & Chromosome location \\
\hline BCL3 & B-cell CLL/lymphoma 3 & $19 \mathrm{q} 13.1-\mathrm{q} 13.2$ \\
\hline BLOC1S1 & Biogenesis of lysosomal organelles complex-1, subunit 1 & $12 \mathrm{q} 13-\mathrm{q} 14$ \\
\hline HESX1 & HESX homeobox 1 & $3 \mathrm{p} 14.3$ \\
\hline IRF2 & Interferon regulatory factor 2 & $4 \mathrm{q} 34.1-\mathrm{q} 35.1$ \\
\hline IRF9 & Interferon regulatory factor 9 & $14 \mathrm{q} 11.2$ \\
\hline JUND & Jun D proto-oncogene & $19 \mathrm{p} 13.2$ \\
\hline LITAF & Lipopolysaccharide-induced TNF factor & $16 \mathrm{p} 13.13$ \\
\hline MIER1 & Mesoderm induction early response 1 , transcriptional regulator & $1 \mathrm{p} 31.3$ \\
\hline MXD1 & MAX dimerization protein 1 & $2 \mathrm{p} 13-\mathrm{p} 12$ \\
\hline NFKBID & $\begin{array}{l}\text { Nuclear factor of kappa light polypeptide gene enhancer in B-cells inhibitor, } \\
\text { delta }\end{array}$ & $19 q 13.12$ \\
\hline PHF11 & PHD finger protein 11 & $13 q 14.2$ \\
\hline SP110 & SP110 nuclear body protein & $2 \mathrm{q} 37.1$ \\
\hline SP140 & SP140 nuclear body protein & $2 \mathrm{q} 37.1$ \\
\hline SP140L & SP140 nuclear body protein-like & $2 q 37.1$ \\
\hline STAT1 & Signal transducer and activator of transcription $1,91 \mathrm{kDa}$ & $2 q 32.2$ \\
\hline STAT4 & Signal transducer and activator of transcription 4 & $2 \mathrm{q} 32.2-\mathrm{q} 32.3$ \\
\hline TRIM22 & Tripartite motif containing 22 & $11 \mathrm{p} 15$ \\
\hline TRIM38 & Tripartite motif containing 38 & $6 \mathrm{p} 21.3$ \\
\hline ZNF385A & Zinc finger protein $385 \mathrm{~A}$ & $12 q 13.13$ \\
\hline
\end{tabular}




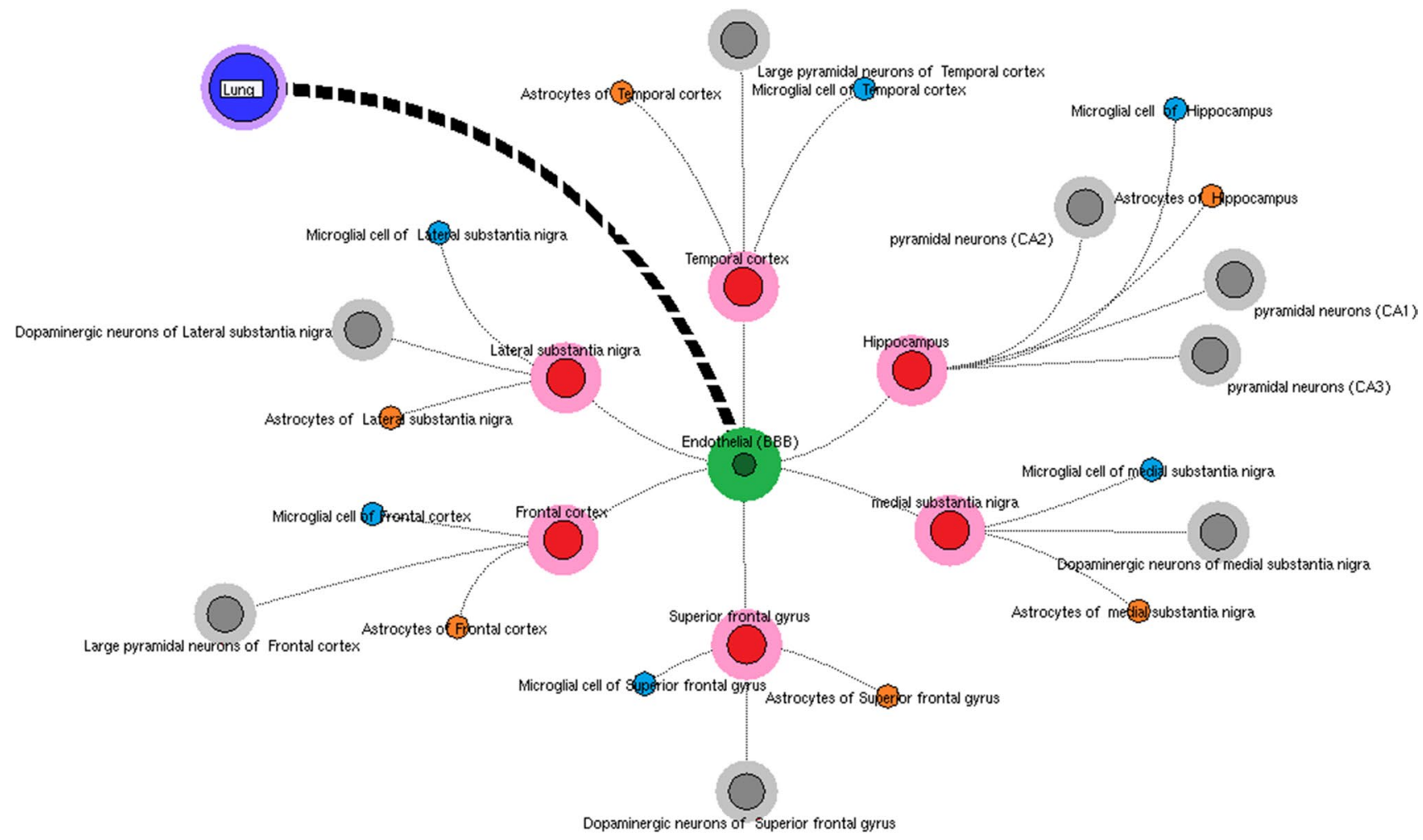

Fig. 1 Exosomal communication network. Network representing the exosomal connectivity between lungs to the neuronal regions associated with neurodegenerative diseases through the blood-brain barrier (BBB). The lungs are represented as violet node defining connec- tion with the BBB (green node) by bold dotted edges. BBB connects various brain regions associated with $\mathrm{PD}$ (yellow) and $\mathrm{AD}$ (red). The gray-colored nodes represent the neurons, orange nodes represent astrocyte, and blue nodes represent microglia cells substantia nigra, STAT1 regulates 19 PD genes, whereas at superior frontal gyrus region, STAT1 regulates 65 genes related to PD. Similarly, in AD, STAT1 regulates 74 AD genes at the temporal cortex. STAT1 regulates 40 genes in the hippocampus, whereas at frontal cortex regions, STAT1 regulates 16 genes related to $\mathrm{AD}$.

\section{Molecular Enrichment}

We investigated the biological process of each regulatory network in PD (Supplementary Material 1) and AD (Supplementary Material 2), which showed dysregulation in the immune response, signal transduction, apoptosis, and response to stress metabolism. Furthermore, the pathway analysis of the PD regulatory network showed regulating TRAIL signaling pathway, estrogen receptor signaling, Alpha9 beta1 integrin signaling events, LKB1 signaling events, ErbB receptor signaling network, Sphingosine 1-phosphate (S1P) pathway, mTOR signaling pathway, internalization of ErbB1, S1P1 pathway, Arf6 trafficking events, and insulin pathway (Fig. 9). Similarly, the AD regulatory networks significantly enriched and contributed to the TRAIL signaling pathway, IL5-mediated signaling events, Syndecan-1 signaling, estrogen receptor signaling, proteoglycan, focal adhesion kinase signaling, urokinasetype plasminogen activator (uPA), and uPAR-mediated signaling (Fig. 10).

\section{Discussion}

The mechanism of COVID-19 causing neurological manifestation is not clearly understood. The current study suggests that COVID-19 accelerates the immune process and oxidative stress causing difficulty in patients with neurodegenerative diseases [45]. The complexity of the neurodegenerative process and the significant involvement of newly emerged pandemic COVID-19 make it necessary to investigate the association of COVID-19 in PD and AD, the most prevalent neurodegenerative diseases worldwide.

In the present study, the complex systems biological framework was implemented that integrates various transcriptome datasets to demonstrate the influence of exosomal transporting molecules from the lung to the brain on COVID-19 infection. During inflammation, lung cells release exosomes that carry the abundance of signaling molecules to avoid cellular stress. We identified 19 transcription factors (LITAF, IRF2, IRF9, PHF11, ZNF385A, MIER1, 
Fig. 2 Co-expressed network of neurodegenerative disease genes with TFs. Network representing the co-expressed up-regulated genes (blue ellipse) in PD and $\mathrm{AD}$ along with the 19 transcription factors (green polygon) transported from the lung through exosome on COVID-19 infection

Fig. 3 Regulatory network in lateral substantia nigra. Network representing the transcription factors (green polygon) regulating (back dotted edges) the up-regulated genes (yellow ellipse) in Parkinson's disease
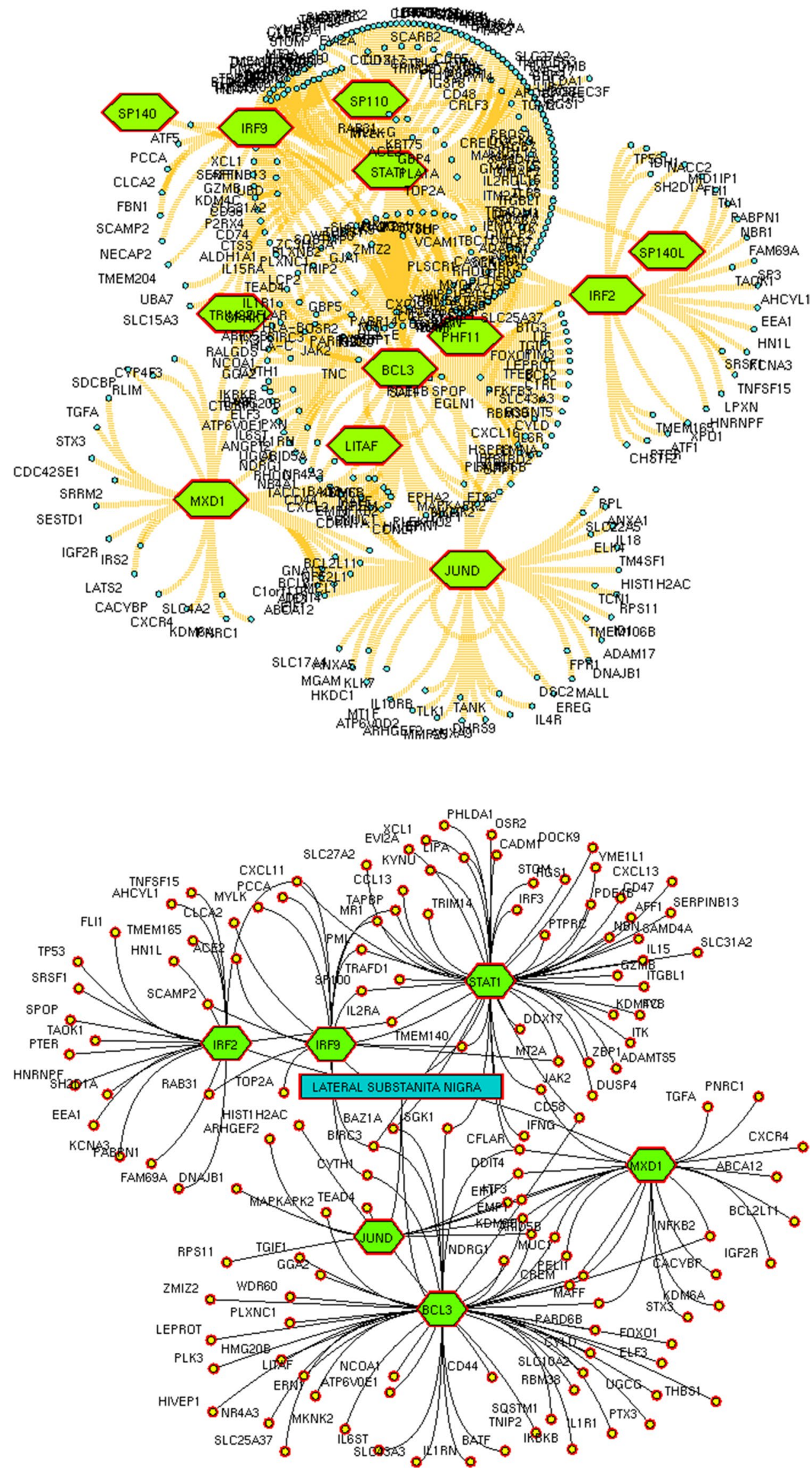
Fig. 4 Regulatory network in medial substantia nigra. Network representing the transcription factors (green polygon) regulating (back dotted edges) the up-regulated genes (yellow ellipse) in Parkinson's disease

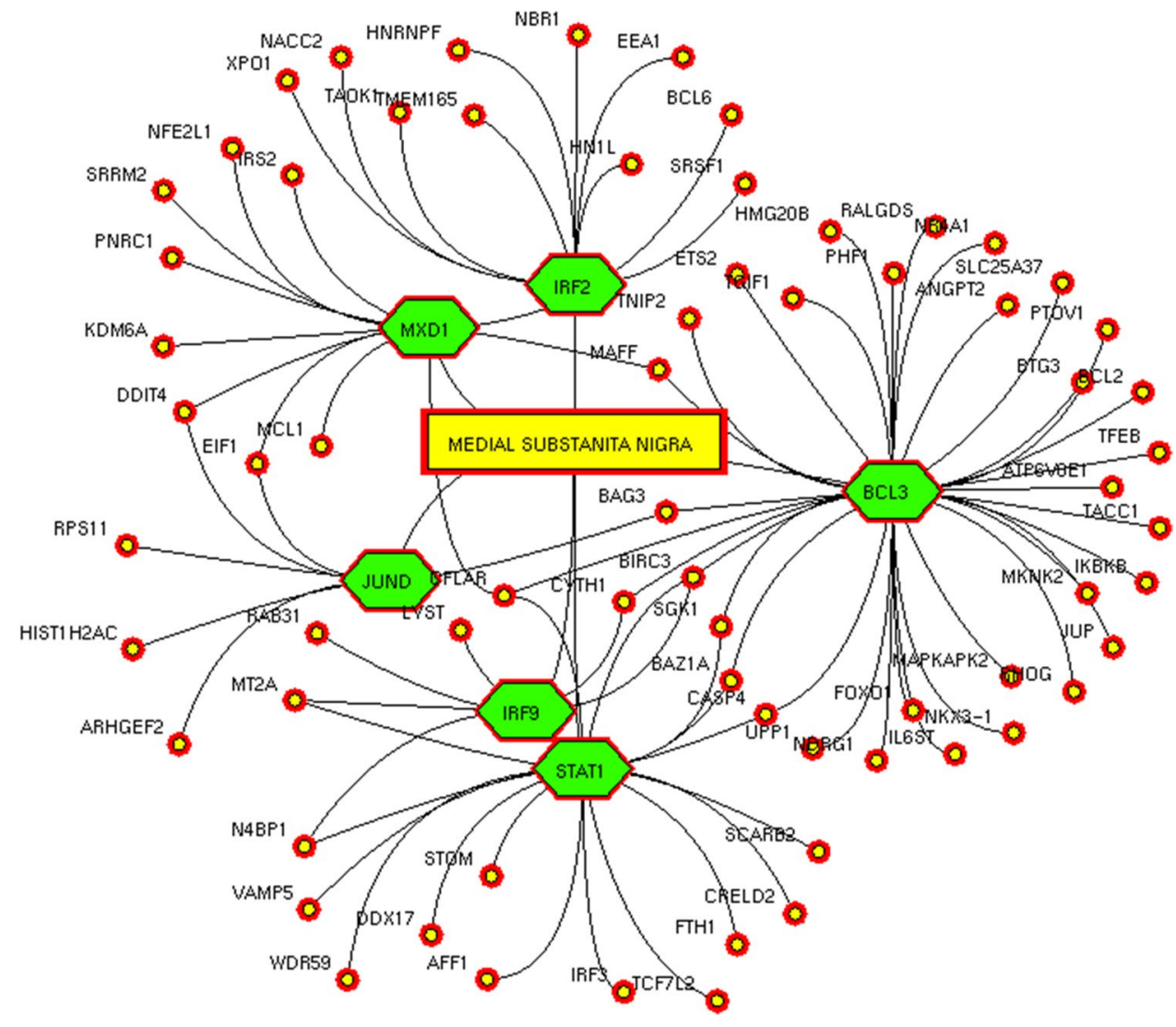

Fig. 5 Regulatory network in the frontal gyrus. Network representing the transcription factors (green polygon) regulating (back dotted edges) the upregulated genes (yellow ellipse) in Parkinson's disease

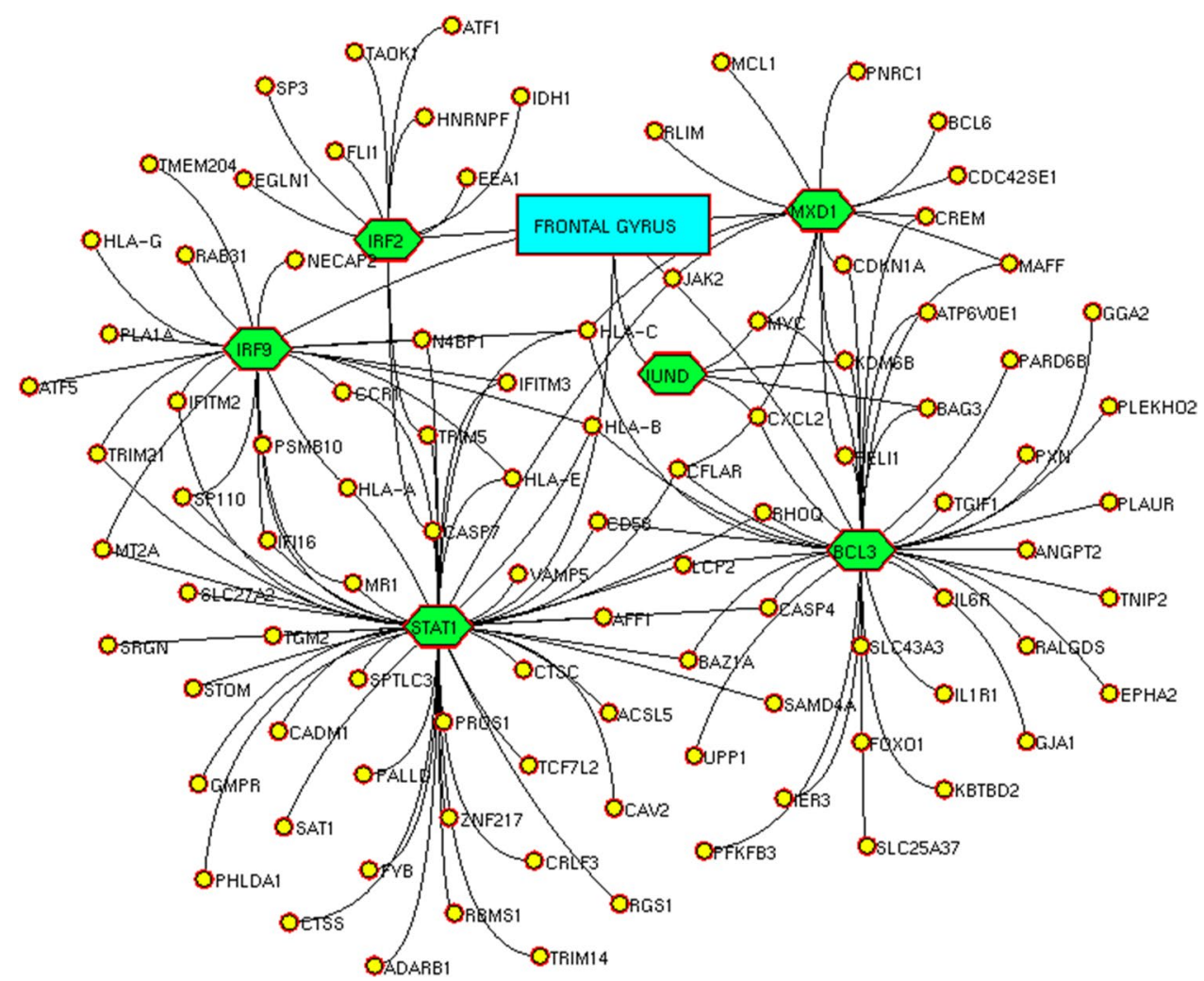


Fig. 6 Regulatory network in the frontal cortex. Network representing the transcription factors (green polygon) regulating (back dotted edges) the upregulated genes (yellow ellipse) in Alzheimer's disease
Fig. 7 Regulatory network in the hippocampus. Network representing the transcription factors (green polygon) regulating (back dotted edges) the upregulated genes (yellow ellipse) in Alzheimer's disease
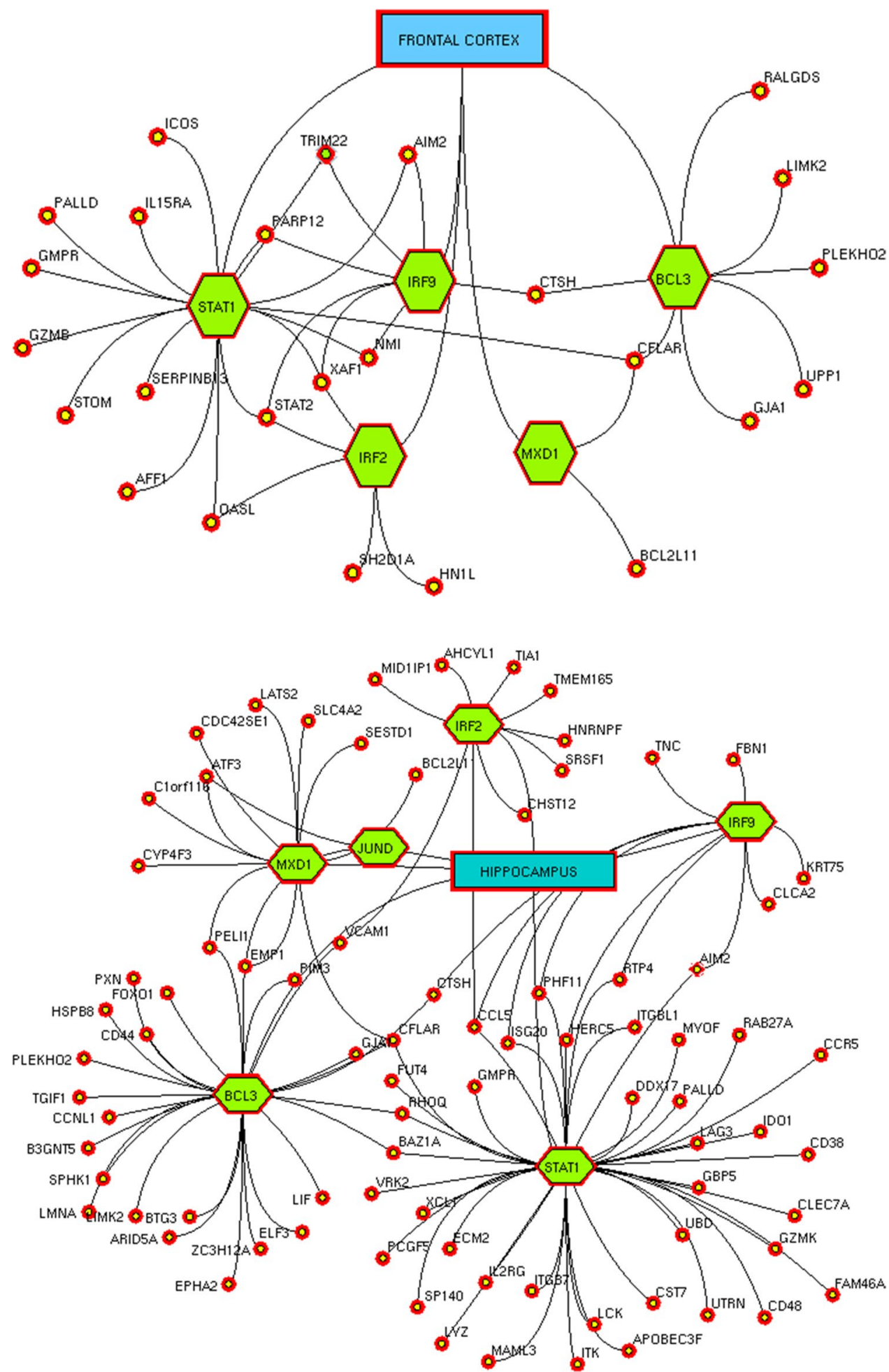

SP140L, BCL3, STAT4, NFKBID, TRIM22, JUND, STAT1, BLOC1S1, SP110, TRIM38, MXD1, SP140, and HESX1) encoding mRNA over-represented on COVID-19 infection in lungs. Most of these transcription factors play a vital role in the host viral defense mechanism. Mainly, IRF2 and IRF9 are members of the interferon (IFN) regulatory factors involved in regulating interferon system with TRIM22 and STAT1 as anti-viral and anti-bacterial components [46, 47]. The IRF9 regulates inflammation and immune cell in autoimmune disease [48-50]. TRIM22 is the component of TRIM family involved in apoptosis, immune signaling, and antiviral activities [50]. Interestingly, TRIM22 restricts the 


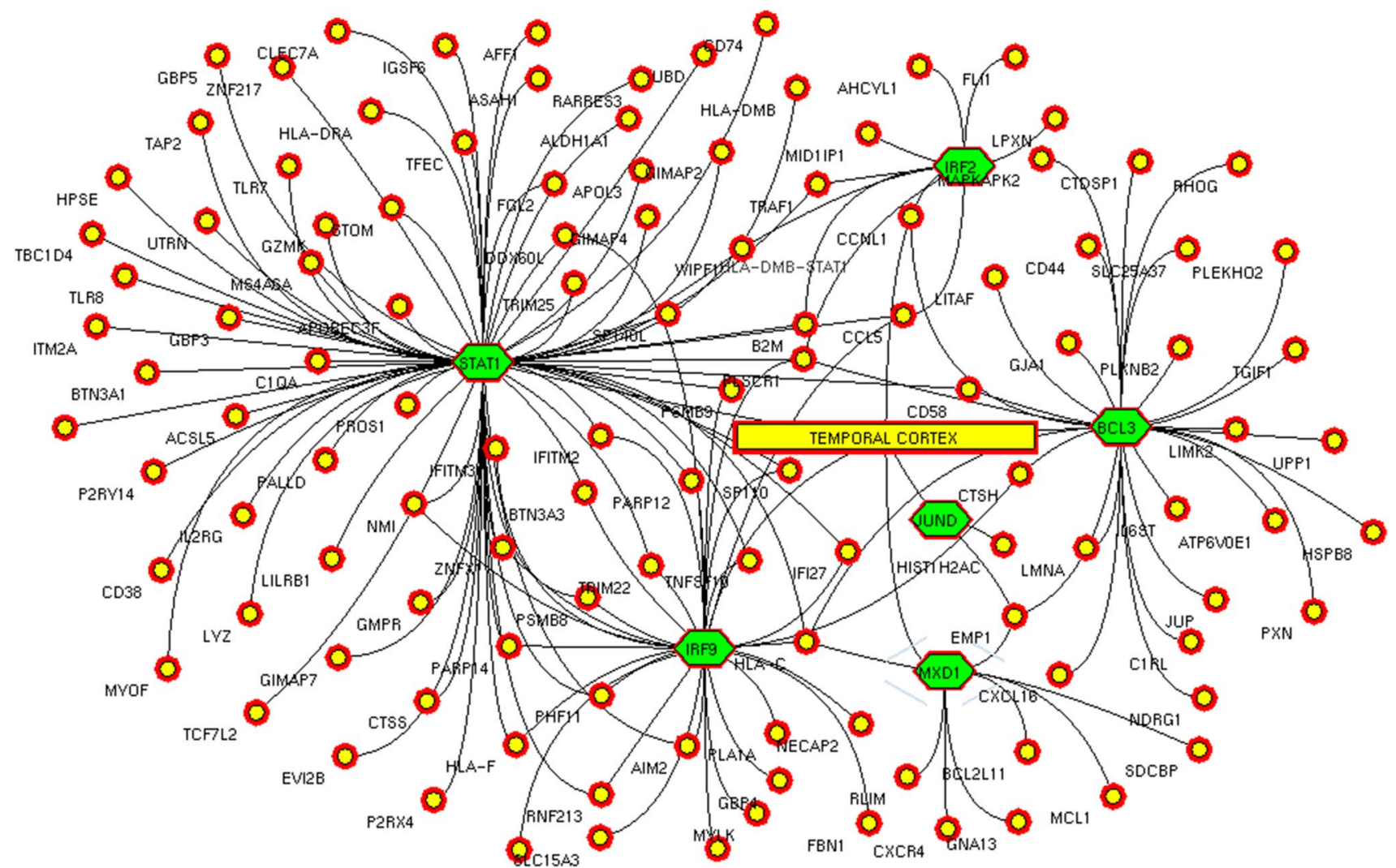

Fig. 8 Regulatory network in the temporal cortex. Network representing the transcription factors (green polygon) regulating (back dotted edges) the up-regulated genes (yellow ellipse) in Alzheimer's disease

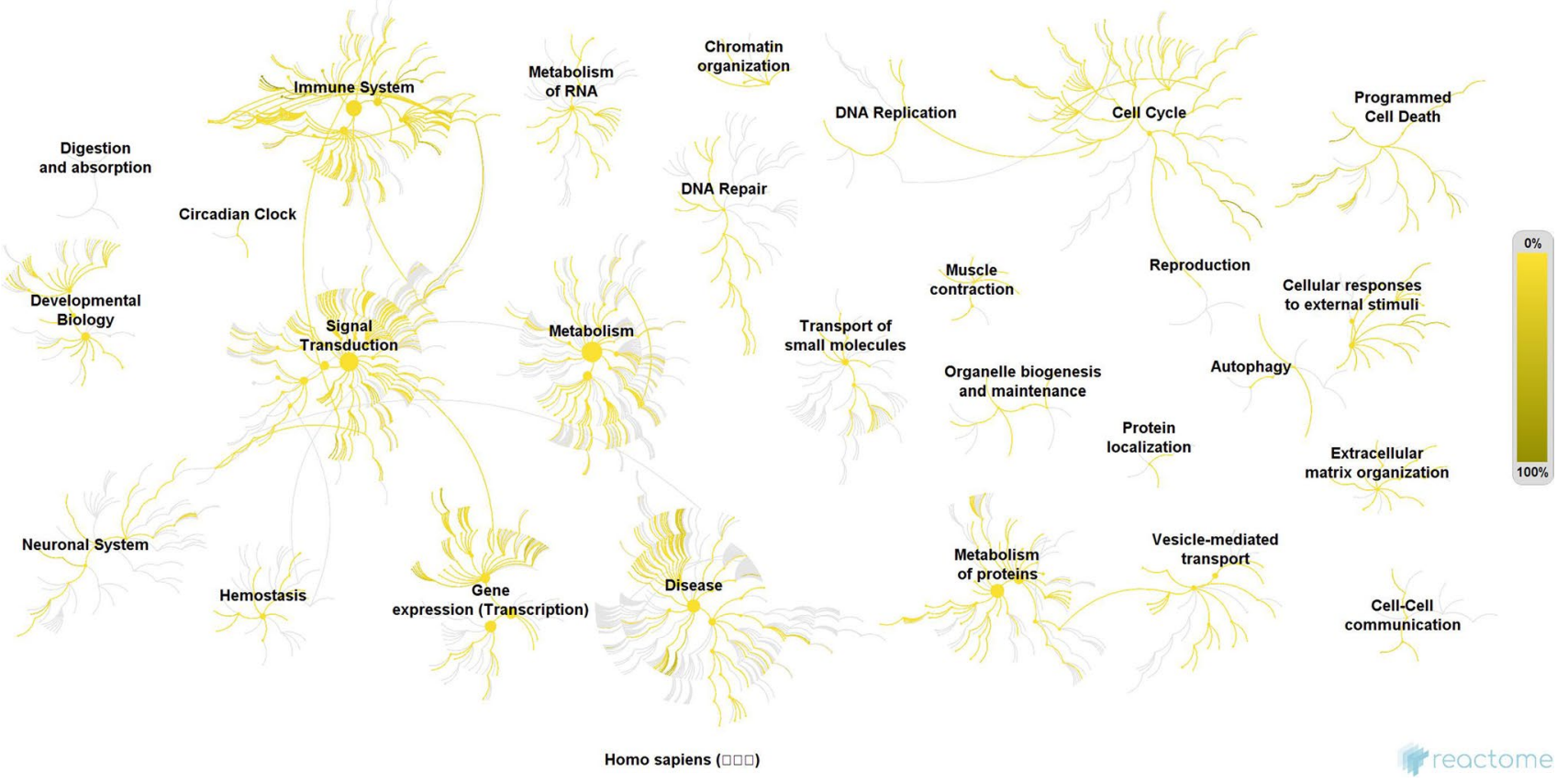

Fig. 9 Functional enrichment of Parkinson's disease genes regulated by the transcription factors. Transcription factors activating PD genes showing major involvement in the immune system, signal transduction, cell cycle, and programmed cell death 


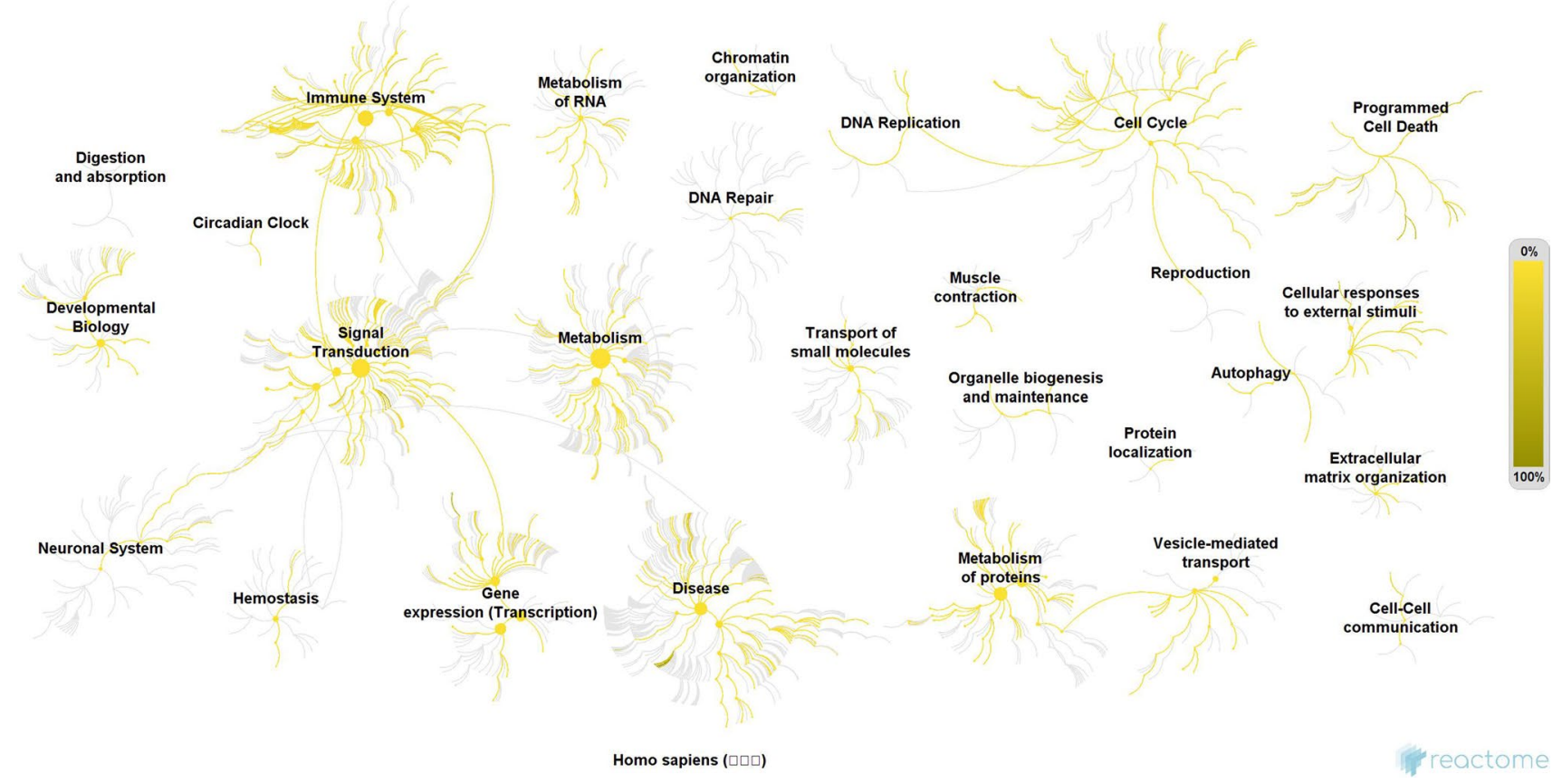

Fig. 10 Functional enrichment of Alzheimer's disease genes regulated by the transcription factors. Transcription factors activating AD genes showing significant involvement in the immune system, signal transduction, metabolism, cell cycle, and response to external stimuli

replication of various viruses, including HIV, hepatitis B virus, and influenza A virus [51-53]. Likewise, MXD1 is the MAX dimerization protein 1 transcription factor regulating cell differentiation, proliferation, and apoptosis. MXD1 was noticed up-regulated in $\mathrm{CD} 14^{+}$monocytes and $\mathrm{CD}^{+}{ }^{+} \mathrm{T}$ cells on COVID-19 infection [54]. Recently, Mamoor et al. demonstrate the role of MXD1 in host cells' response to COVID19 infection [55]. Also, Howard et al. reported the influence of MXD1 expression upon H1N1 and H9N2/G1 viral infection in the host [56]. Furthermore, silencing of MXD1 decreases the H9N2 replication process [56]. Similarly, PHF11 is one of the interferon-stimulated genes expressed by a host on viral infections that regulates cytokine genes in T lymphocytes [57, 58]. A study reports enriched cytokines and chemokines in HIV-infected patients' exosomes compared to HIV-negative control [59]. Similarly, lipopolysaccharide (LPS) stimulated RAW 264.7 cells to produce exosomes with increased chemokine and RNA molecules involved in regulating inflammation [60].

Herein, our RNA-Seq analysis of the GSE121307 dataset showed 19 TFs in the lung-derived exosomes on COVID19 infection. The lung exosome interacts with brain microvascular endothelial cells that anatomically connect the pathological hotspot of neurodegenerative diseases like $\mathrm{AD}$ and PD. The exosomal connectivity between the lungs to $\mathrm{BBB}$ and $\mathrm{BBB}$ to other neuronal regions was determined based on literature [27-32]. Exosomal delivery of 19 TFs encoding mRNAs to the neuronal hotspot may get translated into proteins using translational machinery of target cells. Timothy et al. report the functional activity of translated exosome-shuttled mRNA in the target cells [61]. Similarly, Montecalvo et al. and Corrado et al. reported the change in molecular pathways in the target cells on the transfer of exosomal cargoes [62, 63]. Also, Valadi et al. report the cellular modulation in target cell on translating the exosomal mRNA into proteins by the target cell translational machinery [64]. Particularly, Valadi et al. demonstrated the support of translational machinery for the protein synthesis of exosome-transported mRNAs in the target cells using in vitro translation assay [64].

In the present study, we demonstrated that the translation of these 19 transcription factors will activate their target genes. Among these, six TFs were noticed activating 469 genes associated with $\mathrm{AD}$ and $\mathrm{PD}$ on binding to their regulatory regions. Most of these activated genes are involved in inflammatory, apoptosis, and other signal transduction processes that contribute to the neurodegeneration process. Although our study identified novel information about the transcription factors regulating neurodegenerative genes on COVID-19 infection, few limitations need to be considered. First, the identified transcriptional regulatory networks were derived using a heterogeneous dataset. Second, no functional exploration was made to understand the significant regulatory network based on COVID-19 infection. Third, difference in regulatory behavior of the network based on gender was not established due to limited samples in dataset that 
avoid sub-group analysis. Alternatively, the strength of this study need to be acknowledged: (1) This study presents a novel approach of integrating multiple datasets to understand the pathogenesis caused by exosome communication in COVID-19. (2) To the best of knowledge, this is the first study to present a unique exosome communication model that aids in understanding the impact of peripheral exosome on the CNS.

\section{Conclusions}

In conclusion, our study explores the behavior of exosomal TF regulating PD and AD causative genes at neuronal regions on SARS-CoV-2 infection. Although the public repository data were used, we implemented several curation levels to achieve the pathologically relevant TF regulatory network associated with neurodegenerative diseases. More particularly, the step-wise procedure implemented in the present study was strategically confirmed at each stage of methodology with biological and literature evidence. With the rapidly enlarging COVID-19 pandemic, there is an unprecedented level of impact on the research sector and access to laboratory work. Therefore, many research investigations are underway to utilize the publicly available data on SARS-CoV-2 to expand the research efforts to handle the present critical situation. On this basis, the present study enhances the knowledge of COVID-19 influencing neuronal pathogenesis that describes the previously unknown mechanism of the COVID-19 pandemic. However, our hypothesis warrants further investigation.

Supplementary Information The online version contains supplementary material available at https://doi.org/10.1007/s12035-021-02485-9.

Acknowledgements All authors thank their institute for the support and encouragements.

Author Contribution S.S.S.J.A.: conceptualization, investigation, writing—original draft. P.P., M.K., A.S., R.M.: investigation. S.R.: manuscript draft correction.

Data Availability The datasets GSE121307, GSE8397, and GSE36980 used in this study are available online at NCBI (https://www.ncbi.nlm. nih.gov/).

\section{Declarations}

Ethics Declarations Not applicable

Consent to Participate Not applicable

Consent for Publication The manuscript contains no individual person's data in any form.

Competing Interests The authors declare no competing interests.

\section{References}

1. Colombo M, Raposo G, Théry C (2014) Biogenesis, secretion, and intercellular interactions of exosomes and other extracellular vesicles. Annu Rev Cell Dev Biol 30:255-289

2. Statello L, Maugeri M, Garre E, Nawaz M, Wahlgren J, Papadimitriou A, Lundqvist C, Lindfors L et al (2018) Identification of RNA-binding proteins in exosomes capable of interacting with different types of RNA: RBP-facilitated transport of RNAs into exosomes. PLoS One. 13(4):e0195969

3. Johnstone RM, Adam M, Hammond JR, Orr L, Turbide C (1987) Vesicle formation during reticulocyte maturation. Association of plasma membrane activities with released vesicles (exosomes). J Biol Chem 262(19):9412-20

4. Ludwig N, Whiteside TL, Reichert TE (2019) Challenges in exosome isolation and analysis in health and disease. Int J Mol Sci 20(19):4684

5. Zhao W, Zheng XL, Zhao SP (2015) Exosome and its roles in cardiovascular diseases. Heart Fail Rev 20(3):337-348

6. Mathews PM, Levy E (2019) Exosome production is key to neuronal endosomal pathway integrity in neurodegenerative diseases. Front Neurosci 12(13):1347

7. Schorey JS, Cheng Y, Singh PP, Smith VL (2015) Exosomes and other extracellular vesicles in host-pathogen interactions. EMBO Rep 16(1):24-43

8. Liu Z, Zhang X, Yu Q, He JJ (2014) Exosome-associated hepatitis $\mathrm{C}$ virus in cell cultures and patient plasma. Biochem Biophys Res Commun 455(3-4):218-222

9. Fleming A, Sampey G, Chung MC, Bailey C, van Hoek ML, Kashanchi F, Hakami RM (2014) The carrying pigeons of the cell: exosomes and their role in infectious diseases caused by human pathogens. Pathog Dis 71(2):109-120

10. Ahmed SSSJ, Paramasivam P, Raj K, Kumar V, Murugesan R, Ramakrishnan V (2020) Regulatory cross talk between SARS$\mathrm{CoV}-2$ receptor binding and replication machinery in the human host. Front Physiol 30(11):802

11. Barrera FJ, Shekhar S, Wurth R, Moreno-Pena PJ, Ponce OJ, Hajdenberg M, Alvarez-Villalobos NA, Hall JE et al (2020) Prevalence of diabetes and hypertension and their associated risks for poor outcomes in Covid-19 patients. J Endocr Soc. 4(9):bvaa102

12. Renu K, Prasanna PL, Valsala Gopalakrishnan A (2020) Coronaviruses pathogenesis, comorbidities and multi-organ damage - a review. Life Sci. 255:117839

13. Badierah RA, Uversky VN, Redwan EM (2020) Dancing with Trojan horses: an interplay between the extracellular vesicles and viruses. J Biomol Struct Dyn 30:1-27

14. Elrashdy F, Aljaddawi AA, Redwan EM, Uversky VN (2020) On the potential role of exosomes in the COVID-19 reinfection/reactivation opportunity. J Biomol Struct Dyn 9:1-12

15. Qian Z, Travanty EA, Oko L, Edeen K, Berglund A, Wang J, Ito Y, Holmes KV et al (2013) Innate immune response of human alveolar type II cells infected with severe acute respiratory syndromecoronavirus. Am J Respir Cell Mol Biol 48(6):742-748

16. Müller JA, Groß R, Conzelmann C, Krüger J, Merle U, Steinhart J, Weil T, Koepke L et al (2021) SARS-CoV-2 infects and replicates in cells of the human endocrine and exocrine pancreas. Nat Metab 3(2):149-165

17. Su H, Yang M, Wan C, Yi LX, Tang F, Zhu HY, Yi F, Yang $\mathrm{HC}$ et al (2020) Renal histopathological analysis of 26 postmortem findings of patients with COVID-19 in China. Kidney Int 98(1):219-227

18. Mao L, Jin H, Wang M, Hu Y, Chen S, He Q, Chang J, Hong C et al (2020) Neurologic manifestations of hospitalized patients with coronavirus disease 2019 in Wuhan, China. JAMA Neurol 77(6):683-690 
19. Ritchie K, Chan D, Watermeyer T (2020) The cognitive consequences of the COVID-19 epidemic: collateral damage? Brain Commun. 2(2):fcaa069

20. Singh AK, Bhushan B, Maurya A, Mishra G, Singh SK, Awasthi R (2020) Novel coronavirus disease 2019 (COVID-19) and neurodegenerative disorders. Dermatol Ther. 33(4):e13591

21. Pavel A, Murray DK, Stoessl AJ (2020) COVID-19 and selective vulnerability to Parkinson's disease. Lancet Neurol 19(9):719

22. Ousset PJ, Vellas B (2020) Viewpoint: Impact of the Covid-19 outbreak on the clinical and research activities of memory clinics: an Alzheimer's disease center facing the Covid-19 crisis. J Prev Alzheimers Dis 7(3):197-198

23. Brown EG, Chahine LM, Goldman SM, Korell M, Mann E, Kinel DR, Arnedo V, Marek KL et al (2020) The effect of the COVID19 pandemic on people with Parkinson's disease. J Parkinsons Dis 10(4):1365-1377

24. Naughton SX, Raval U, Pasinetti GM (2020) Potential novel role of COVID-19 in Alzheimer's disease and preventative mitigation strategies. J Alzheimers Dis 76(1):21-25

25. Heneka MT, Golenbock D, Latz E, Morgan D, Brown R (2020) Immediate and long-term consequences of COVID-19 infections for the development of neurological disease. Alzheimer's Res Ther 12:1-3

26. Tan DBA, Armitage J, Teo TH, Ong NE, Shin H, Moodley YP (2017) Elevated levels of circulating exosome in COPD patients are associated with systemic inflammation. Respir Med 132:261-264

27. Wahlund CJ, Eklund A, Grunewald J, Gabrielsson S (2017) Pulmonary extracellular vesicles as mediators of local and systemic inflammation. Front Cell Dev Biol 26(5):39

28. Hermanns MI, Unger RE, Kehe K, Peters K, Kirkpatrick CJ (2004) Lung epithelial cell lines in coculture with human pulmonary microvascular endothelial cells: development of an alveolocapillary barrier in vitro. Lab Invest 84(6):736-752

29. Zhang Y, Liu D, Chen X, Li J, Li L, Bian Z, Sun F, Lu J et al (2010) Secreted monocytic miR-150 enhances targeted endothelial cell migration. Mol Cell 39(1):133-144

30. Chen HX, Liang FC, Gu P, Xu BL, Xu HJ, Wang WT, Hou JY, Xie DX et al (2020) Exosomes derived from mesenchymal stem cells repair a Parkinson's disease model by inducing autophagy. Cell Death Dis 11(4):1-7

31. Schiera G, Di Liegro CM, Di Liegro I (2015) Extracellular membrane vesicles as vehicles for brain cell-to-cell interactions in physiological as well as pathological conditions. Biomed Res Int 2015:152926

32. Cui GH, Guo HD, Li H, Zhai Y, Gong ZB, Wu J, Liu JS, Dong YR et al (2019) RVG-modified exosomes derived from mesenchymal stem cells rescue memory deficits by regulating inflammatory responses in a mouse model of Alzheimer's disease. Immun Ageing 16(1):1-2

33. Elliott RO, He M (2021) Unlocking the power of exosomes for crossing biological barriers in drug delivery. Pharmaceutics 13(1): 122

34. Saint-Pol J, Gosselet F, Duban-Deweer S, Pottiez G, Karamanos Y (2020) Targeting and crossing the blood-brain barrier with extracellular vesicles. Cells 9(4):851

35. Reynolds JL, Mahajan SD (2021) SARS-COV2 alters blood brain barrier integrity contributing to neuro-inflammation. J Neuroimmune Pharmacol 16(1):4-6

36. Chen CC, Liu L, Ma F, Wong CW, Guo XE, Chacko JV, Farhoodi HP, Zhang SX et al (2016) Elucidation of exosome migration across the blood-brain barrier model in vitro. Cell Mol Bioeng 9(4):509-529. https://doi.org/10.1007/s12195-016-0458-3
37. Matsumoto J, Stewart T, Banks WA, Zhang J (2017) The transport mechanism of extracellular vesicles at the blood-brain barrier. Curr Pharm Des 23(40):6206-6214

38. Blanco-Melo D, Nilsson-Payant BE, Liu WC, Uhl S, Hoagland D, Møller R, Jordan TX, Oishi K et al (2020) Imbalanced host response to SARS-CoV-2 drives development of COVID-19. Cell 181(5):1036-1045.e9

39. Genschmer KR, Russell DW, Lal C, Szul T, Bratcher PE, Noerager BD, Abdul Roda M, Xu X et al (2019) Activated PMN exosomes: pathogenic entities causing matrix destruction and disease in the lung. Cell 176(1-2):113-126.e15

40. Moran LB, Duke DC, Deprez M, Dexter DT, Pearce RK, Graeber MB (2006) Whole genome expression profiling of the medial and lateral substantia nigra in Parkinson's disease. Neurogenetics $7(1): 1-11$

41. Hokama M, Oka S, Leon J, Ninomiya T, Honda H, Sasaki K, Iwaki T, Ohara T et al (2014) Altered expression of diabetesrelated genes in Alzheimer's disease brains: the Hisayama study. Cereb Cortex 24(9):2476-2488

42. Verfaillie A, Imrichová H, Van de Sande B, Standaert L, Christiaens V, Hulselmans G, Herten K, Sanchez MN et al (2014) iRegulon: from a gene list to a gene regulatory network using large motif and track collections. PLoSComput Biol. 10(7):e1003731

43. Guan D, Tian H (2017) Integrated network analysis to explore the key genes regulated by parathyroid hormone receptor 1 in osteosarcoma. World J Surg Oncol 15(1):177

44. Maere S, Heymans K, Kuiper M (2005) BiNGO: a Cytoscape plugin to assess overrepresentation of gene ontology categories in biological networks. Bioinformatics 21(16):3448-3449

45. Ntyonga-Pono MP (2020) COVID-19 infection and oxidative stress: an under-explored approach for prevention and treatment? Pan Afr Med J 35(Suppl 2):12

46. Gao B, Wang Y, Xu W, Duan Z, Xiong S (2010) A 5' extended IFN-stimulating response element is crucial for IFN-gammainduced tripartite motif 22 expression via interaction with IFN regulatory factor-1. J Immunol 185(4):2314-2323

47. Rauch I, Rosebrock F, Hainzl E, Heider S, Majoros A, Wienerroither S, Strobl B, Stockinger S et al (2015) Noncanonical effects of IRF9 in intestinal inflammation: more than type I and type III interferons. Mol Cell Biol 35(13):2332-2343

48. Huber M, Suprunenko T, Ashhurst T, Marbach F, Raifer H, Wolff S, Strecker T, Viengkhou B et al (2017) IRF9 prevents CD8 ${ }^{+} \mathrm{T}$ cell exhaustion in an extrinsic manner during acute lymphocytic choriomeningitis virus infection. J Virol 91(22):e01219-17.

49. Smith S, Fernando T, Wu PW, Seo J, Gabhann JN, Piskareva O, McCarthy E, Howard D et al (2017) MicroRNA-302d targets IRF9 to regulate the IFN-induced gene expression in SLE. J Autoimmun 1(79):105-111

50. Gao B, Xu W, Wang Y, Zhong L, Xiong S (2013) Induction of TRIM22 by IFN-? involves JAK and PC-PLC/PKC, but not MAPKs and $\mathrm{pI} 3 \mathrm{~K} / \mathrm{Akt} / \mathrm{mTOR}$ pathways. J Interferon Cytokine Res 33(10):578-587

51. Barr SD, Smiley JR, Bushman FD (2008) The interferon response inhibits HIV particle production by induction of TRIM22. PLoS pathog. 4(2):e1000007

52. Gao B, Duan Z, Xu W, Xiong S (2009) Tripartite motif-containing 22 inhibits the activity of hepatitis B virus core promoter, which is dependent on nuclear-located RING domain. Hepatology 50(2):424-433

53. Di Pietro A, Kajaste-Rudnitski A, Oteiza A, Nicora L, Towers GJ, Mechti N, Vicenzi E (2013) TRIM22 inhibits influenza A virus infection by targeting the viral nucleoprotein for degradation. $\mathrm{J}$ Virol 87(8):4523-4533

54. Johri S, Jain D, Gupta I (2020) Integrated analysis of bulk multi omic and single-cell sequencing data confirms the molecular 
origin of hemodynamic changes in Covid-19 infection explaining coagulopathy and higher geriatric mortality. medRxiv. 2020.04.26.20081182

55. Mamoor S (2020) The MAX dimerization protein MXD1 is differentially expressed and transcriptionally induced in multiple models of coronavirus infection. OSF Preprints. https://doi.org/ 10.31219/osf.io/8unvq

56 Yim HCH, Leon TYY, Li JCB (2020) MXD1 regulates the $\mathrm{H} 9 \mathrm{~N} 2$ and H1N1 influenza A virus-induced chemokine expression and their replications in human macrophage. J Leukoc Biol. 108(5):1631-40

57. Clarke E, Rahman N, Page N, Rolph MS, Stewart GJ, Jones GJ (2008) Functional characterization of the atopy-associated gene PHF11. J Allergy Clin Immunol 121(5):1148-1154

58. Rahman N, Stewart G, Jones G (2010) A role for the atopy-associated gene PHF11 in T-cell activation and viability. Immunol Cell Bio 88(8):817-824

59. Konadu KA, Chu J, Huang MB, Amancha PK, Armstrong W, Powell MD, Villinger F, Bond VC (2015) Association of cytokines with exosomes in the plasma of HIV-1-seropositive individuals. J Infect Dis 211(11):1712-1716

60. McDonald MK, Tian Y, Qureshi RA, Gormley M, Ertel A, Gao R, Lopez EA, Alexander GM et al (2014) Functional significance of macrophage-derived exosomes in inflammation and pain. Pain 155(8):1527-1539
61. Ung TH, Madsen HJ, Hellwinkel JE, Lencioni AM, Graner MW (2014) Exosome proteomics reveals transcriptional regulator proteins with potential to mediate downstream pathways. Cancer Sci 105(11):1384-1392

62. Montecalvo A, Larregina AT, Shufesky WJ, Stolz DB, Sullivan ML, Karlsson JM, Baty CJ, Gibson GA et al (2012) Mechanism of transfer of functional microRNAs between mouse dendritic cells via exosomes. Blood 119(3):756-766

63. Corrado C, Raimondo S, Chiesi A, Ciccia F, De Leo G, Alessandro R (2013) Exosomes as intercellular signaling organelles involved in health and disease: basic science and clinical applications. Int J Mol Sci 14(3):5338-5366

64. Valadi H, Ekström K, Bossios A, Sjöstrand M, Lee JJ, Lötvall JO (2007) Exosome-mediated transfer of mRNAs and microRNAs is a novel mechanism of genetic exchange between cells. Nat Cell Biol 9(6):654-659

Publisher's Note Springer Nature remains neutral with regard to jurisdictional claims in published maps and institutional affiliations. 